\title{
Nonlinear speed and yaw control for wind turbine powered vessels
}

Proc IMechE Part I:

J Systems and Control Engineering

I-II

(c) IMechE 2016

Reprints and permissions:

sagepub.co.uk/journalsPermissions.nav DOI: 10.1 I77/095965।815623709

pii.sagepub.com

(SAGE

\section{Alexander Stotsky}

\begin{abstract}
The application of conventional wind turbines on board a vessel creates a number of new challenges for turbine control systems. Thrust force is used in marine applications as an additional control variable for propulsion of the vessel. Variability of the wind speed and wind direction, together with the yaw rate and range constraints, impose strong requirements on the turbine control system. A new projection algorithm that projects the turbine thrust force on the heading direction of vessel is proposed in this paper for controlling propulsion. Variations in the wind direction and wind speed are counteracted via turbine yaw angle, making the turbine thrust force always aligned with the heading direction of the vessel. The conventional $K \omega^{2}$ speed controller is modified for varying yaw offset and a combined algorithm for simultaneous control of the turbine speed and thrust force is proposed. Stability of the $K \omega^{2}$ speed controller for varying yaw offset is proved via the Lyapunov method. The controller also takes into account the constraints on the yaw rate and minimizes the turbine gyroscopic effects via a proper choice of the virtual upper bound of the input voltage of the yaw motor. All of the results are illustrated by simulations using measurement data acquired from the Hönö turbine.
\end{abstract}

\section{Keywords}

Wind turbine on vessel, thrust force as propulsion, Lyapunov stability of $K \omega^{2}$ speed controller with varying yaw offset, projection algorithm, nonlinear yaw control with bounded input, minimization of turbine gyroscopic effects

Date received: 10 March 20I5; accepted: I5 November 2015

\section{Introduction}

Wind power available at sea motivates the use of wind turbines on board vessels for both electricity generation and propulsion. This idea has attracted significant attention and research efforts in recent years. ${ }^{1-3}$ Conventional wind power systems need to be modified for marine applications to take into account a number of challenges to the control systems. Conventional wind turbines are designed and controlled for maximum power output with certain constraints on mechanical loads. ${ }^{4,5}$ Marine applications also include propulsion of the ship using the thrust force created by the turbine, in addition to electricity generation. Variability of the wind speed and wind direction imposes new requirements for turbine control systems, and necessitates the development of novel control architectures, which are suitable for use in the marine environment. Adaptation of conventional turbine control systems to marine applications meets the following challenges/problems, which are described and solved in this paper.

(1) The first problem is a definition of controllability of the turbine thrust force as a control variable.
In this paper, the controllability of the turbine thrust force is associated with invertibility of the thrust force model, where the thrust force is modeled as a function of the yaw offset and the wind speed. The model is calibrated using turbine data obtained from the Hönö turbine (see Figure 1 and Table 1).

(2) The second problem is modification of the conventional turbine speed $K \omega^{2}$ controller for varying yaw offset. Yaw offset that controls the turbine thrust force has a direct impact on the performance of the speed controller. In this paper, this controller is modified by taking into account the yaw offset. Moreover, stability of the speed control system is proved via the Lyapunov method and the performance is quantified by simulations using measurement data.

\section{Chalmers Industriteknik, Sweden}

\section{Corresponding author:}

Alexander Stotsky, Chalmers Industriteknik, Chalmers Teknikpark, Sven Hultins gata 9, SE-4I 288 Gothenburg, Sweden.

Email: alexander.stotsky@chalmers.se 


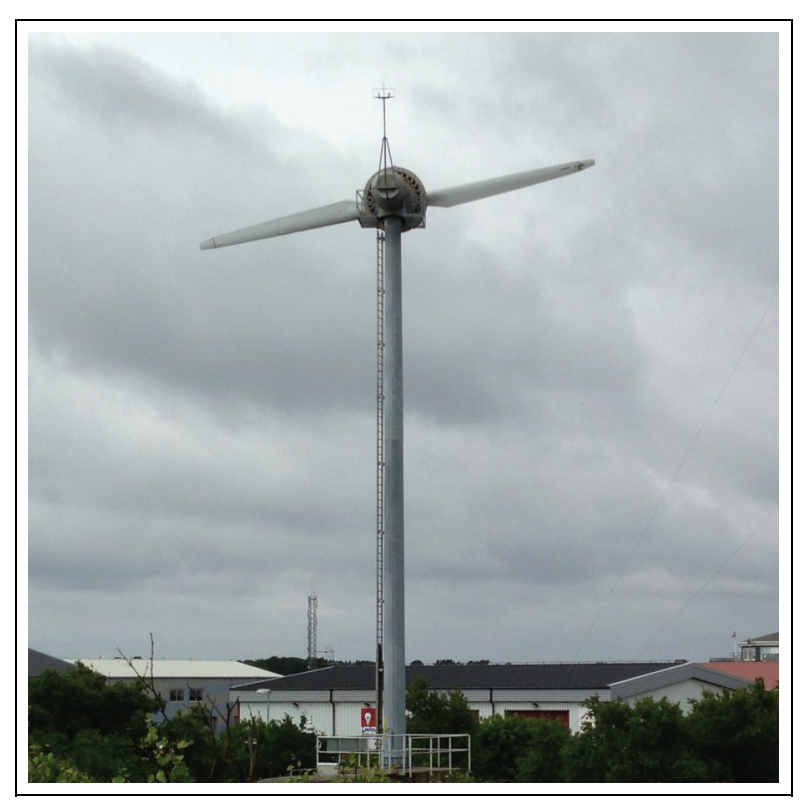

Figure I. The Hönö turbine located outside Gothenburg, Sweden. The turbine has two blades with rotor diameter $13.5 \mathrm{~m}$ and power rating $35 \mathrm{~kW}$. The turbine parameters are summarized in Table I.

Table I. Parameters of the Hönö turbine.

\begin{tabular}{lll}
\hline Parameters & & \\
\hline Parameter name & Value & Unit \\
\hline Number of blades & 2 & - \\
Mass of the blade & 157.6 & $\mathrm{~kg}$ \\
Rotor radius, $R$ & 6.75 & $\mathrm{M}$ \\
Maximal rotational speed, $\omega_{\max }$ & 75 & $\mathrm{rpm}$ \\
Rated power & 35 & $\mathrm{~kW}$ \\
Rated Wind Speed & 10 & $\mathrm{~m} / \mathrm{s}$ \\
Optimal tip-speed ratio, $\lambda_{*}$ & 8 & - \\
Rotor inertia, J & $8 \times 10^{3}$ & $\mathrm{~kg} \mathrm{~m} \mathrm{~m}^{2}=\mathrm{N} \mathrm{m} \mathrm{s}^{2}$ \\
Gear ratio, $N$ & $\mathrm{I}$ & - \\
\hline
\end{tabular}

(3) The third problem is to design a coordinated control system that controls both turbine speed and thrust force. A simple and easy-to-implement combined control strategy is proposed in this paper for controlling the turbine thrust force via the yaw offset and turbine speed. The control architecture is realized via a projection algorithm that projects the turbine force onto the heading direction of vessel. Variations in the wind direction and in the wind speed are compensated via turbine yaw angle, making the turbine thrust force always aligned with the heading direction of the vessel. Moreover, a method for minimization of the turbine gyroscopic effects by limiting the yaw rate by control is also proposed. The yaw rate is limited via a proper choice of the virtual upper bound of the input voltage of the DC yaw motor. All results are confirmed by simulations using measurement data acquired from the Hönö turbine.

The paper is organized as follows. The model of the turbine thrust force as a control variable is described in the next section. The concept of controllability of the thrust force is introduced in Section 3. The conventional $K \omega^{2}$ speed controller is modified for varying yaw offset in Section 4. Turbine yaw system modeling and control are presented in Section 5. Simultaneous speed and thrust force control, which realizes the projection method, is presented in Section 6. The paper ends with some concluding remarks in Section 7.

\section{Data-driven modeling of the thrust force as a control variable}

The model of the thrust force as a control variable, which is based on measurement data acquired from the experimental wind turbine (located on Hönö outside Gothenburg, Sweden; see Figure 1), is created for identification of the propulsion controllability of the turbine. ${ }^{6}$ The turbine parameters are summarized in Table 1.

This two-bladed turbine, operated at different yaw offsets, was used for identification of the thrust force. The thrust was measured by two pairs of strain gauge sensors, placed at a distance of $1900 \mathrm{~mm}$ above the tower foundation flange. The yaw offset is used as an input and the resulting thrust force was treated as an output of the turbine.

Determination of the resulting thrust force direction and amplitude on the Hönö turbine is shown in Figure 2. Two strain gauge sensors are installed in a south-westerly and north-westerly direction forming an orthogonal coordinate system for calculation of the direction and the amplitude of the resulting thrust force. The deviations of the resulting thrust force from the yaw direction are associated with misalignment between wind and yaw directions.

The two misalignments are defined as

$$
\begin{aligned}
& \varphi_{w y}=\varphi_{\text {wind }}-\varphi_{\text {yaw }} \\
& \varphi_{w f}=\varphi_{\text {wind }}-\varphi_{\text {force }}
\end{aligned}
$$

where $\varphi_{w y}$ is wind-to-yaw misalignment (turbine yaw offset), which is defined as the difference between the wind direction $\varphi_{\text {wind }}$ and the position of the nacelle $\varphi_{\text {yaw }}$ and $\varphi_{w f}$ is the difference between wind direction and direction of the resulting thrust force $\varphi_{\text {force }}$ (i.e. windto-thrust misalignment). Notice that the wind direction, the yaw angle and the thrust direction are given with respect to true north according to the nautical reference system (see Figure 2).

The model, which defines the relationship between the mean values of wind-to-yaw misalignment, wind speed and the mean values of wind-to-thrust misalignment, can be calibrated using the experimental set-up shown in Figure 2. Measurements acquired on the 


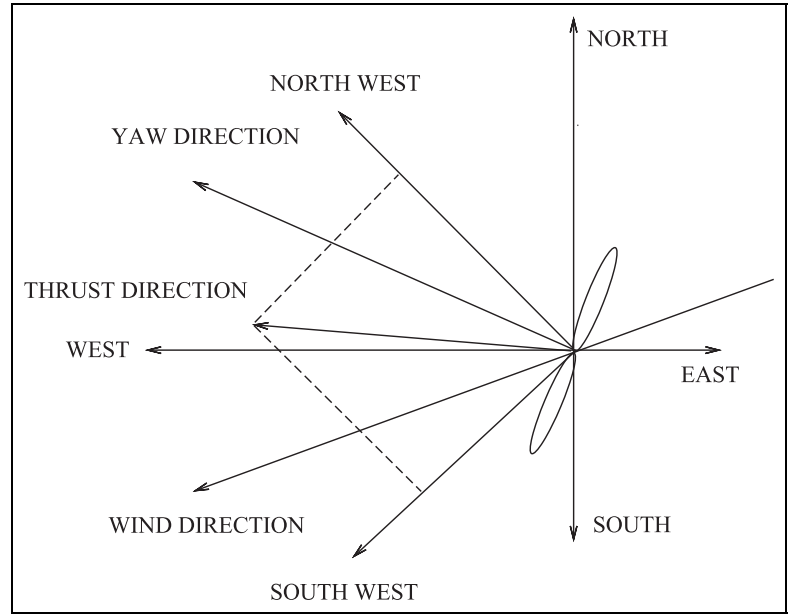

Figure 2. Determination of the resulting thrust force direction and amplitude on the Hönö turbine. Two strain gauge sensors are installed in a south-westerly and north-westerly direction forming a local orthogonal coordinate system for calculation of the direction and the amplitude of the resulting thrust force. The angle between the north-west axis and the thrust direction and the amplitude of the thrust force are calculated as $\arctan \left(\frac{s}{n}\right)$ and $\sqrt{n^{2}+s^{2}}$, respectively, in the local reference system, where $n$ and $s$ are the components measured in the north-westerly and south-westerly directions, respectively. All of the angles are defined with respect to true north in the nautical reference system.

Hönö turbine at different wind speeds for a number of turbine yaw angles, with respect to fixed wind directions, are the inputs to this model validation. The outputs of two strain gauge sensors, which were installed in south-westerly and north-westerly directions, were averaged over the time intervals for constant average values of wind-to-yaw misalignment and wind speed. The number of points which represent average values of wind-to-thrust misalignment as a function of windto-yaw misalignment and wind speed were sufficiently large to fit the surface, which, in turn, can be seen as a data-driven model of the thrust force. This model can be written in the form

$$
\varphi_{w f}=f\left(\varphi_{w y}, V\right)
$$

where wind-to-thrust misalignment $\varphi_{w f}$ is the output variable, and wind-to-yaw misalignment $\varphi_{w y}$ and the wind speed $V$ are the two input variables.

Note that motion of the vessel should be accounted for in model (3) for marine applications. The relative wind vector is calculated using the parallelogram law by adding the wind vector and the vessel motion vector. This vector determines the relative wind speed $V$, as an input to the function (3), and the relative wind direction $\varphi_{\text {wind }}$, in (1) and (2).

The model in (3) is presented as a surface in three dimensional space in Figure 3, where all of the variables are given with normalized units. The amplitude of the thrust force as a function of the same input variables was calibrated in the same way. These models allow

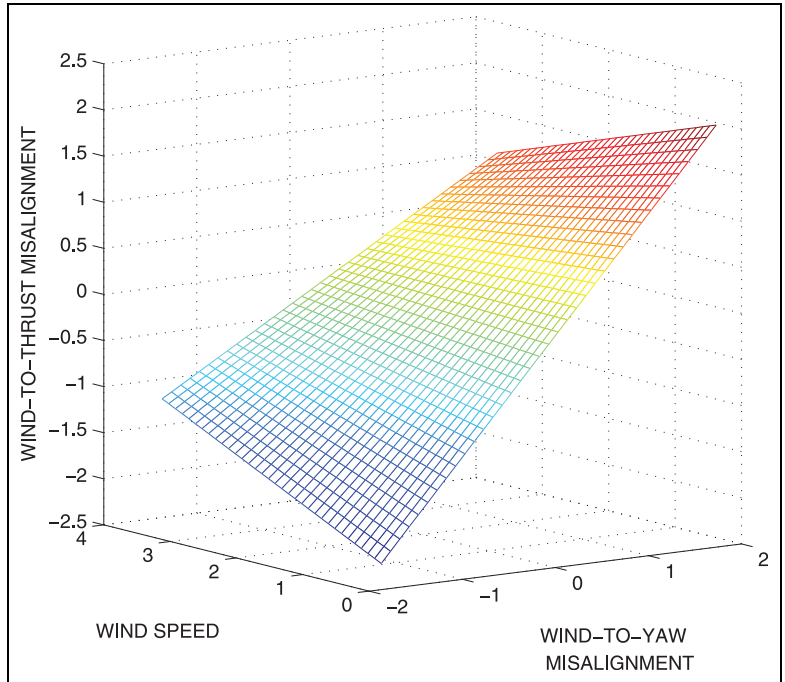

Figure 3. The model of the thrust force $\varphi_{w f}=f\left(\varphi_{w y}, V\right)$ is presented as a surface in three-dimensional space, where windto-thrust misalignment $\varphi_{w f}$ is the output variable, and wind-toyaw misalignment $\varphi_{\text {wy }}$ and the wind speed $V$ are two input variables. All of the variables are presented in normalized units.

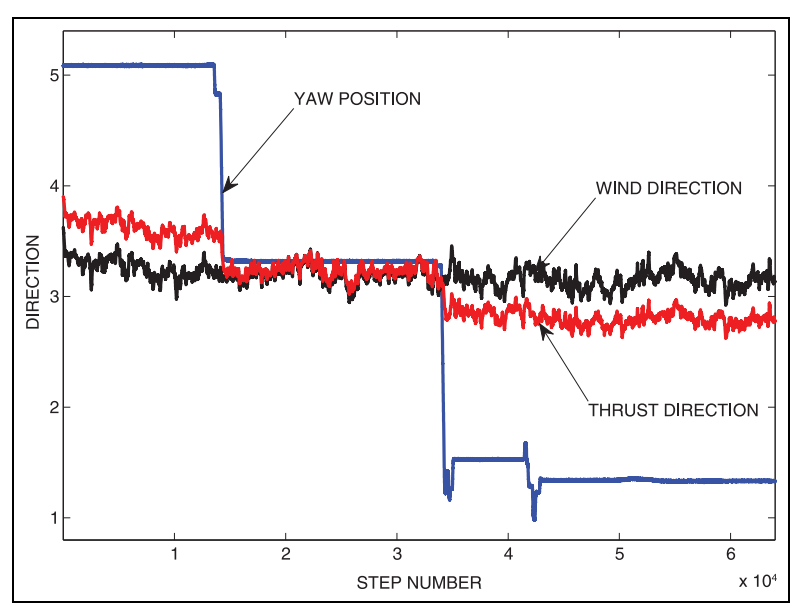

Figure 4. The direction of the thrust force is controlled on the Hönö turbine by changing yaw position. It is shown that windto-yaw misalignment implies wind-to-thrust misalignment. Wind direction is plotted with a black line, turbine yaw position is plotted with a blue line and, finally, the direction of the thrust force is plotted with a red line. The thrust direction coincides with the wind direction, when wind-to-yaw misalignment is close to zero. Yaw position and wind direction are measured on the Hönö turbine, and the direction of the thrust force is calculated using the model presented in Figure 3. All of the variables are presented with normalized units.

monitoring of both the direction and amplitude of the turbine thrust force.

The direction of the thrust force can be controlled by changing the yaw position as illustrated in Figure 4. The figure shows that wind-to-yaw misalignment implies wind-to-thrust misalignment, and that thrust force can be controlled via yaw offset. Wind direction is plotted with a black line, turbine yaw position is 
plotted with a blue line and, finally, the direction of the thrust force is plotted with a red line.

Note that the model given in (3) can be improved using measurements of the thrust force on board the vessel. Only a few measurement points obtained on board the vessel can be used for adaptation of the surface presented in Figure 3, using the surface adaptation method described by Stotsky. ${ }^{7}$

\section{Control of the turbine thrust force}

Figure 4 shows that the turbine thrust force can be controlled via yaw position at different wind speeds. The turbine thrust force controllability is directly associated with the model given in (3), which relates wind-to-thrust misalignment with wind-to-yaw misalignment at different values of the wind speed. The turbine thrust force is controllable if wind-to-yaw misalignment can be calculated as a function of wind-to-thrust misalignment. It means that, for a given thrust force direction (with respect to the wind direction), there exists a unique turbine yaw position that corresponds to this thrust direction. The formal definition of controllability of the thrust force is given below.

Definition of controllability of the turbine thrust force. The turbine thrust force is controllable, if there exists an inverse function $f^{-1}(\cdot)$, where $f$ is defined in (3) such that

$$
\varphi_{w y}=f^{-1}\left(\varphi_{w f}, V\right)
$$

and the relationship

$$
\varphi_{w f}=f\left(\varphi_{w y}, V\right)=f(\underbrace{f^{-1}\left(\varphi_{w f}, V\right)}_{=\varphi_{w y}}, V)=\varphi_{w f}
$$

holds according to the definition of the inverse function. The inverse function, which has wind-to-thrust misalignment and wind speed as input variables and wind-to-yaw misalignment as the output variable, can be presented as an inverse surface to the surface shown in Figure 3. Such an inverse surface is presented in Figure 5.

Equation (4), presented in the form of look-up table, as shown in Figure 5, can be used as the control algorithm, which calculates wind-to-yaw misalignment as a function of desired wind-to-thrust misalignment, for a certain wind speed. Use of the inverse model (4) as a control is verified using measurement data as follows. First, the thrust force direction is calculated for a certain trajectory of the actual yaw position, as shown in Figure 4. Then the yaw position is calculated via windto-yaw misalignment, which, in turn, is calculated via the inverse model (4) with two inputs: (1) the thrust force direction calculated in the first step and (2) the wind speed. The model is inverted correctly if the actual yaw position is close to the yaw position calculated via the inverse model (4). The performance of the inversion technique is illustrated in Figure 6, where the desired

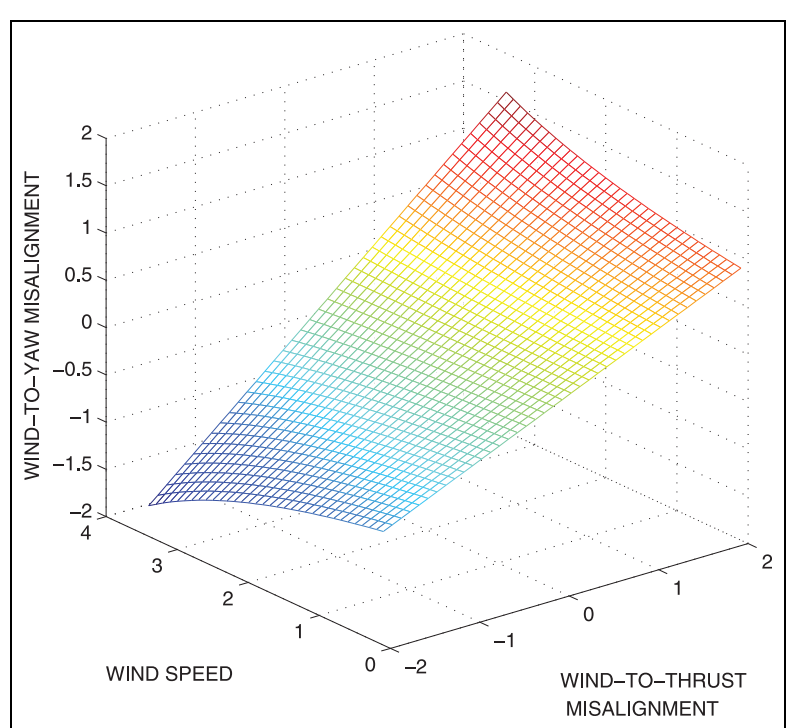

Figure 5. The inverse function $\varphi_{w y}=f^{-1}\left(\varphi_{w f}, V\right)$, which has the wind-to-thrust misalignment and the wind speed as the input variables and the wind-to-yaw misalignment as the output variable, is presented as a surface in three-dimensional space. All of the variables are presented with normalized units.

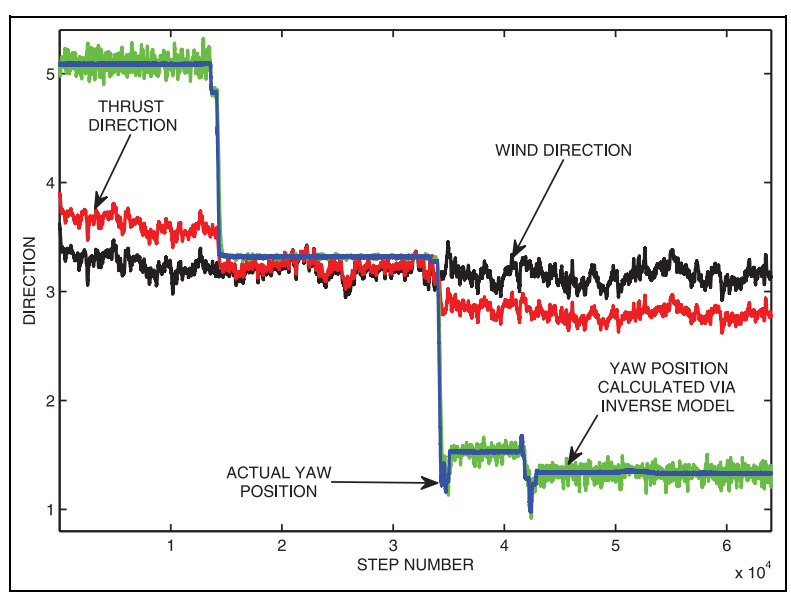

Figure 6. Verification of the performance of the model inversion technique. The desired direction of the thrust force is plotted with a red line. Yaw position, which creates deviation between wind direction and thrust force direction, is plotted with a blue line. The desired yaw position calculated using the inverse model is plotted with a green line. The difference between the actual and the desired yaw positions quantifies the control performance. All of the variables are presented with normalized units.

direction of the thrust force is plotted with a red line. The yaw position, which creates deviation between wind direction and thrust force direction, is plotted with a blue line. Finally, yaw position, which is calculated using the inverse model, is plotted with a green line. The difference between the actual and calculated yaw positions quantifies the performance of the control strategy based on the model inversion technique, described above. 


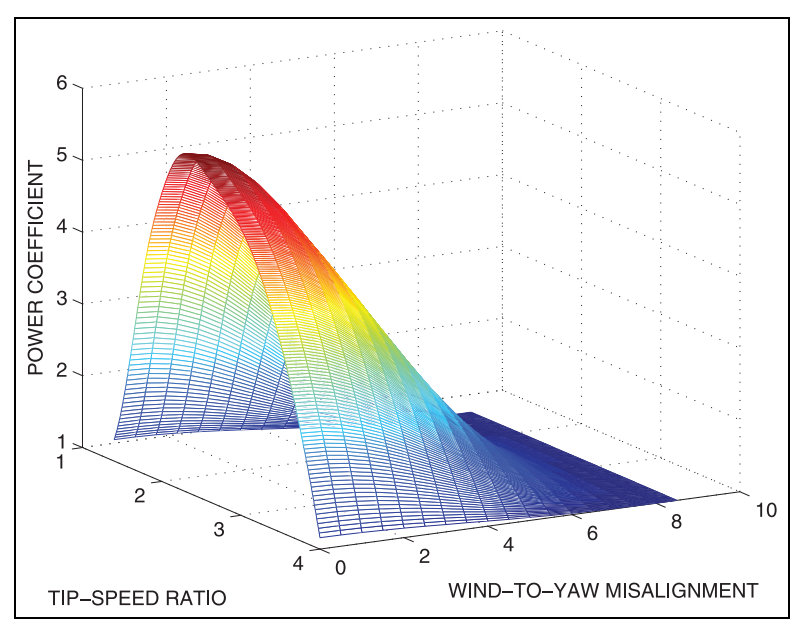

Figure 7. Power coefficient $C_{p}\left(\lambda, \varphi_{w y}\right)=\frac{P_{r}}{P_{\text {wind }}}$ which depends on the wind-to-yaw misalignment $\varphi_{w y}$ and the tip-speed ratio $\lambda=\frac{\omega R}{V}$. All of the variables are presented with normalized units.

\section{Turbine speed control in the presence of yaw offset}

\section{Aerodynamic model}

The wind turbine converts energy from the wind to the rotor shaft that rotates at a speed $\omega$. The power of the wind available in the swept area $A=\pi R^{2}$ (where $R$ is the rotor radius) is given by $P_{\text {wind }}=\frac{1}{2} \rho A V^{3}$ and depends on the wind speed $V$ and air density $\rho$. Note that the swept area $A$ is defined for perfect alignment of the turbine rotational axis with the wind. Yaw misalignment has a direct impact on the swept area, ${ }^{8}$ and this impact is accounted for in the power coefficient, ${ }^{9}$ which relates the power of the rotor $P_{r}$ and the wind power $C_{p}\left(\lambda, \varphi_{w y}\right)=\frac{P_{r}}{P_{\text {wind }}}$ (see Figure 7) which, in turn, depends on the wind-to-yaw misalignment $\varphi_{w y}$ and the tip-speed ratio $\lambda=\frac{\omega R}{V}$ : that is

$$
P_{r}=P_{w i n d} C_{p}\left(\lambda, \varphi_{w y}\right)=\frac{A \rho V^{3} C_{p}\left(\lambda, \varphi_{w y}\right)}{2}
$$
by

The aerodynamic torque applied to the rotor is given

$$
T_{a}=\frac{P_{r}}{\omega}=\frac{A \rho V^{3} C_{p}\left(\lambda, \varphi_{w y}\right)}{2 \omega}
$$

Turbine rotational dynamics can be described using a one-mass model as

$$
J \dot{\omega}=\frac{P_{r}}{N \omega}-T_{g}, \omega_{g}=N \omega
$$

where $\omega_{g}$ is a generator speed, $T_{g}$ is a generator torque, $J$ is a lumped rotational inertia of the system, and $N$ is a gear ratio. The parameters of the Hönö turbine are presented in Table 1, and the nomenclature of the turbine

\begin{tabular}{|c|c|}
\hline \multicolumn{2}{|l|}{ Nomenclature } \\
\hline Variable name & Unit \\
\hline \multicolumn{2}{|l|}{ Aerodynamic and turbine variables } \\
\hline Wind speed, $V$ & $\mathrm{~m} / \mathrm{s}$ \\
\hline Rotor radius, $R$ & $\mathrm{~m}$ \\
\hline Swept area, $A$ & $\mathrm{~m}^{2}$ \\
\hline Air density, $\rho$ & $\mathrm{kg} / \mathrm{m}^{3}$ \\
\hline Wind power, $P_{\text {wind }}$ & W \\
\hline Rotor power, $P_{r}$ & 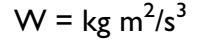 \\
\hline Tip-speed ratio, $\lambda$ & - \\
\hline Optimal tip-speed ratio, $\lambda_{*}$ & - \\
\hline Power coefficient, $C_{p}\left(\lambda, \varphi_{w y}\right)$ & - \\
\hline Maximum power coefficient, $C_{p \max }\left(\varphi_{w y}\right)$ & - \\
\hline Aerodynamic torque, $T_{a}$ & $\mathrm{Nm}=\mathrm{kg} \mathrm{m} / \mathrm{s}^{2}$ \\
\hline Wind direction, $\varphi_{\text {wind }}$ & degrees \\
\hline Position of nacelle, $\varphi_{\text {vaw }}$ & degrees \\
\hline Thrust force direction, $\varphi_{\text {force }}$ & degrees \\
\hline Wind-to-yaw misalignment (yaw offset), $\varphi_{w v}$ & degrees \\
\hline Wind-to-thrust misalignment, $\varphi_{w f}$ & degrees \\
\hline \multicolumn{2}{|l|}{ Driveline variables } \\
\hline Rotor speed, $\omega$ & $\mathrm{rad} / \mathrm{s}$ \\
\hline Generator speed, $\omega_{g} \quad \lambda, V$ & $\mathrm{rad} / \mathrm{s}$ \\
\hline Desired rotor speed, $\omega_{d}=\frac{n_{*} v}{R}$ & $\mathrm{rad} / \mathrm{s}$ \\
\hline Rotor inertia, $J \quad R$ & $\mathrm{~kg} \mathrm{~m}^{2}$ \\
\hline Gear ratio, $N$ & - \\
\hline Generator torque, $T_{g}$ & $\mathrm{Nm}$ \\
\hline
\end{tabular}
model can be found in Table 2 .
Table 2. Nomenclature for the turbine model.

\section{Parameter varying $K \omega^{2}$ turbine speed controller: scheduling of coefficient $K$ as a function of yaw offset}

Feedforward $K \omega^{2}$ controller can be described as

$$
T_{g}=K \omega^{2}, K=\frac{1}{2 N} \rho \pi R^{5} \frac{C_{p \max }\left(\varphi_{w y}\right)}{\lambda_{*}^{3}}
$$

where $C_{p \max }\left(\varphi_{w y}\right)$ is the maximum power coefficient achievable by the turbine with yaw offset $\varphi_{w y}, \lambda_{*}=\frac{\omega_{d} R}{V}$ is the tip-speed ratio (which is independent of yaw offset) at this maximum power coefficient, and $\omega_{d}$ is a desired turbine speed corresponding to $\lambda_{*}$ for a given wind speed $V$.

Note that the controller (9) requires measurements of the turbine speed and yaw misalignment and does not require wind speed measurements. Note, also, that the coefficient $K$ is constant in the classical $K \omega^{2}$ control$\mathrm{ler}^{4}$ and the controller in equation (9) is the parametervarying controller, where the coefficient $K=K\left(\varphi_{w y}\right)$ varies with the yaw offset $\varphi_{w y}$, which can be seen as a parameter.

The aerodynamic torque can be written as

$$
\begin{aligned}
& T_{a}=\frac{1}{2 \omega} \rho A V^{3} C_{p}\left(\lambda, \varphi_{w y}\right)=\frac{\omega^{2}}{2} \rho \pi R^{5} \frac{V^{3}}{R^{3} \omega^{3}} C_{p}\left(\lambda, \varphi_{w y}\right) \\
& =\frac{1}{2} \rho \pi R^{5} \frac{C_{p}\left(\lambda, \varphi_{w y}\right)}{\lambda^{3}} \omega^{2}
\end{aligned}
$$




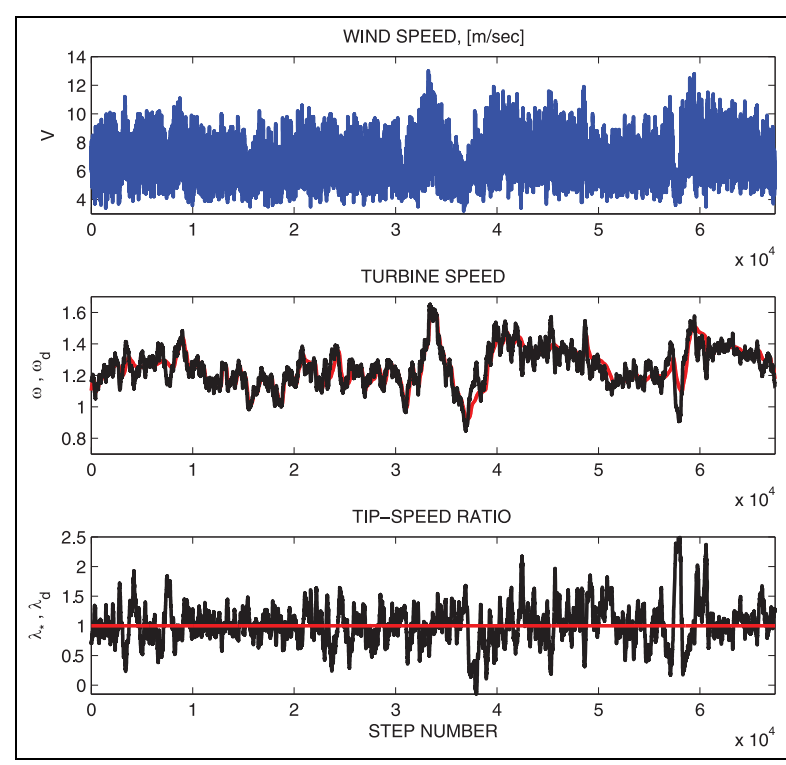

Figure 8. Evaluation of the turbine speed $K \omega^{2}$ controller as given in equation (9). Wind speed is plotted in the first subplot. Actual and desired turbine speeds are plotted with red and black lines, respectively, in the second subplot. Actual and desired tip-speed ratios are plotted with black and red lines, respectively, in the third subplot. Tracking performance is evaluated in the presence of time-varying wind-to-yaw misalignment and is plotted in Figure II. All of the variables, except for wind speed, are presented with normalized units.

The stability of the closed loop system given in equations (8), (9) and (10) is proved in Appendix 1 and it is based on the error model

$$
J \dot{\omega}=\frac{1}{2 N} \rho \pi R^{5} \omega^{2}\left[\frac{C_{p}\left(\lambda, \varphi_{w y}\right)}{\lambda^{3}}-\frac{C_{p \max }\left(\varphi_{w y}\right)}{\lambda_{*}^{3}}\right]
$$

This proof is based on Taylor approximations, which simplify the stability analysis, but the result is valid in only a relatively small neighborhood of the operating point.

The performance of the turbine speed controller given by equation (9) is illustrated in Figure 8, where measured wind speed is plotted in the first subplot, actual and desired turbine speeds are plotted in the second subplot and, finally, tip-speed ratio is plotted in the third subplot. The controller shows a satisfactory performance in the presence of time-varying yaw offset.

\section{Turbine yaw system modeling and control}

\section{Turbine yaw model}

The yaw mechanism of conventional turbines can be divided into two types: passive (free yaw) and active (forced yaw). The passive yaw concept is widely used in small wind turbines. The turbine freely aligns itself to the wind direction by using a tail vane, without wind direction measurement. An active yaw is generally used for larger turbines, equipped with advanced control devices. The yaw mechanism consists of an electric motor and gearbox to keep the turbine aligned with the wind. Rotor position and wind direction are the inputs to the yaw control system, which sends the signals to the yaw electric motor. The control system adjusts the rotor position so as to minimize wind-to-yaw misalignment.

Newton's law, combined with Kirchhoff's law, yields the transfer function of the DC yaw motor given by

$$
H(s)=\frac{G}{s\left(T_{e} s+1\right)\left(T_{m} s+1\right)}
$$

where the yaw angle is the output and the voltage is the input, $T_{m}$ and $T_{e}$ are mechanical and electrical time constants, respectively, $G$ is a DC gain and $s$ is a Laplace variable. The DC yaw motor consists of two subsystems, electrical and mechanical, and the electrical subsystem responds considerably faster than the mechanical subsystem. Therefore, the mechanical subsystem is usually used for the control design, and the influence of the electrical subsystem is neglected. The reduced order model of the DC motor, which accounts for the mechanical subsystem only, can be written as

$$
\frac{\theta}{u_{d c}}=\frac{G}{s\left(T_{m} s+1\right)}
$$

where $\theta$ is the yaw angle and $u_{d c}$ is the voltage, which represents the control signal.

\section{Turbine yaw regulation: minimizing gyroscopic effects via limiting yaw rate by control}

A simple proportional controller, driven by the mismatch between actual and desired yaw angles, makes the closed-loop system stable for both reduced- and full-order models. Significant yaw rate produces gyroscopic effects, which result in large loads on the turbine. The yaw rate should be small enough for gyroscopic effects to be negligible. The turbine yaw motor can operate at a maximum rate in the range $[3,5]$ degrees per second, which is fast enough for the gyroscopic moment to be significant.

Note that gyroscopic loads always occur whenever the turbine is yawing during operation. This results in yaw and tilt moments. It is interesting that the net yaw and tilt moments due to the gyroscopic load are zero and constant, respectively, for a three blade turbine, whereas cyclic yaw and tilt moments appear for a twobladed turbine. However, two-bladed turbines can be more easily demounted to the deck of the vessel in the case of severe weather conditions. Therefore, from this point of view, two-bladed turbines are more suitable for marine applications than three-bladed turbines, but the preference depends on the overall engineering concept .

The yaw rate should be limited to the interval [0.1, 1] degrees per second, which is slow enough to minimize the gyroscopic moment of the turbine. This, in turn, limits the turbine yaw control system. The turbine yaw rate can be limited via limitation of the range of the proportional controller, as shown in Appendix 2. 




Figure 9. Combined turbine feedforward speed control and feedback thrust force control. Generator torque, which realizes the turbine speed control is given by $T_{g}=K \omega^{2}$, where the coefficient $K=\frac{1}{2 N} \rho \pi R^{5} \frac{C_{\text {pmax }}\left(\varphi_{w q}\right)}{\lambda^{3}}$ is scaled according to wind-to-yaw misalignment $\varphi_{w y}$. The feedback control loop, associated with the yaw control, is driven by the inverse model (4), which calculates the desired yaw angle. This angle is the input to the DC motor-based yaw control system (P-regulator with saturation)) that aligns the turbine thrust force with the longitudinal direction of the vessel and minimizes gyroscopic effects. Three signals only are required for implementation of such a control system: turbine speed, wind speed and wind direction. The yaw control system is completely independent of the speed control, whereas the turbine yaw position is used in the speed control system. Unlike conventional turbines, this turbine produces two quantities: power and thrust force.

Note that the yaw controller of the conventional turbines usually consists of proportional, integral and derivative parts. Inclusion of the integral part to the yaw controller, which eliminates steady-state error, is not relevant for turbines with constantly varying yaw position. Inclusion of the derivative part, which improves the transient due to the prediction of the desired trajectory, is not relevant either, due to the essential constraints on the yaw rate. Poor estimation of the performance of the derivative of a rapidly varying wind direction signal is an additional argument for not using the derivative part in the yaw controller.

A simple proportional regulator, which shows robust tracking performance in the presence of variations in wind direction and constraints on the yaw rate, is recommended for this type of application.

\section{Combined nonlinear turbine control: $K \omega^{2}$ speed controller and thrust force projection method}

Unlike conventional turbines, a turbine installed on a vessel has a thrust force, which is used as a propulsion force in addition to the power output (see Figure 9). Note that the turbine thrust force can be used in different ways as a contribution to the propulsion of the vessel. The thrust force controller, described in this section, represents a projection method that projects the vector of the thrust force onto the heading direction of the vessel, so maximizing the contribution of the turbine to the propulsion of the vessel.

The turbine control system, which regulates the two output variables, consists of the turbine feedforward speed control and the turbine feedback yaw control. Generator torque, which realizes the turbine speed feedforward control, is scaled according to wind-toyaw misalignment. The feedback control loop, associated with yaw control, is driven by the inverse model, which calculates the desired yaw angle. This angle is the input to the DC motor-based yaw control system (P-regulator) that aligns the turbine thrust force with the direction in which the vessel is heading. The desired thrust force vector should always be aligned with the heading direction of the vessel for the creation of maximal additional propulsion force. The direction of the desired propulsion force, calculated with respect to the wind direction, is the input to the inverse model, which calculates the desired yaw angle of the turbine. In other words, the turbine thrust force is projected onto the heading direction of the vessel via a proper choice of 


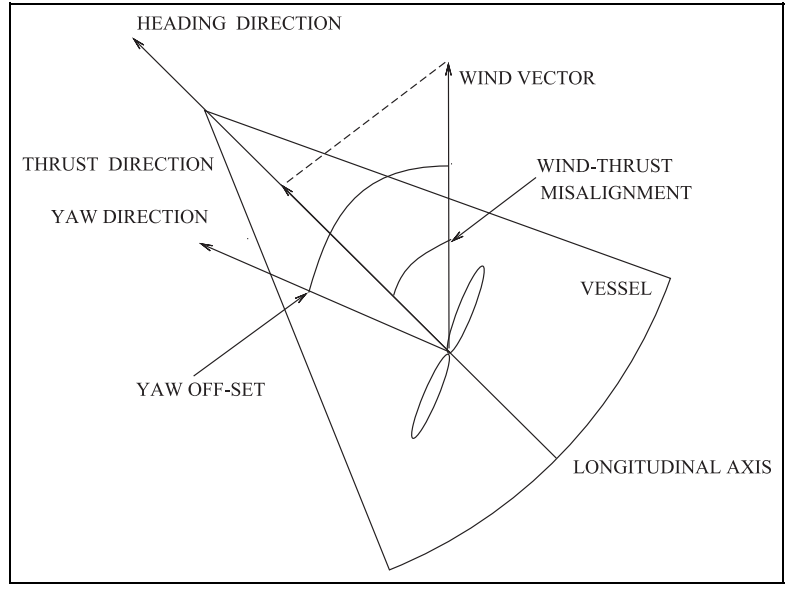

Figure 10. The turbine thrust force projection method. The wind vector applied to the turbine is projected onto the heading direction of the vessel via a properly selected yaw offset. Variations in the wind direction are counteracted via the turbine yaw angle, making the turbine thrust force always aligned with the heading direction of the vessel.

the desired turbine yaw angle, as shown in Figure 10. Notice that the projection strategy may be different during the vessel's maneuvering.

Three signals only are required for implementation of such control system: turbine speed, wind speed and wind direction. The yaw control system is completely independent of the speed control, whereas turbine yaw position is used in the speed control system.

The generator torque $T_{g}$, which realizes the turbine speed control and input voltage $u_{d c}$ to the DC yaw motor, is presented as

$$
\begin{aligned}
& T_{g}=K \omega^{2}, \quad K=\frac{1}{2 N} \rho \pi R^{5} \frac{C_{\text {pmax }}(\theta)}{\lambda_{*}^{3}} \\
& \theta_{d}=f^{-1}\left(\varphi_{w f d}, V\right) \\
& u_{d c}=-k_{p}\left(\theta-\theta_{d}\right), \quad\left|u_{d c}\right| \leqslant \bar{u}, \quad|\theta| \leqslant \bar{\theta}
\end{aligned}
$$

Equation (14) represents generator torque for the feedforward turbine speed control, where all the variables are defined in Parameter varying $K \omega^{2}$ turbine speed controller: Equations (15) and (16) describe the feedback yaw controller with DC voltage $u_{d c}$, which is proportional (with the gain $k_{p}>0$ ) to the mismatch between actual $\theta$ and desired yaw angle $\theta_{d}$, which represents desired wind-to-yaw misalignment. The desired yaw angle is calculated via the inverse model (15), described in equation (4), with the desired wind-tothrust misalignment $\varphi_{w f d}$ and the wind speed $V$ as inputs. The desired wind-to-thrust misalignment is, in turn, calculated using the projection method so that the thrust force is always aligned with the heading direction of the vessel. Notice that the input voltage control of the DC motor is nonlinear due to presence of a nonlinear inverse model (15). Additional nonlinearities are introduced via (a) the control parameter $\bar{u}$, which limits the yaw rate of the turbine for minimization of

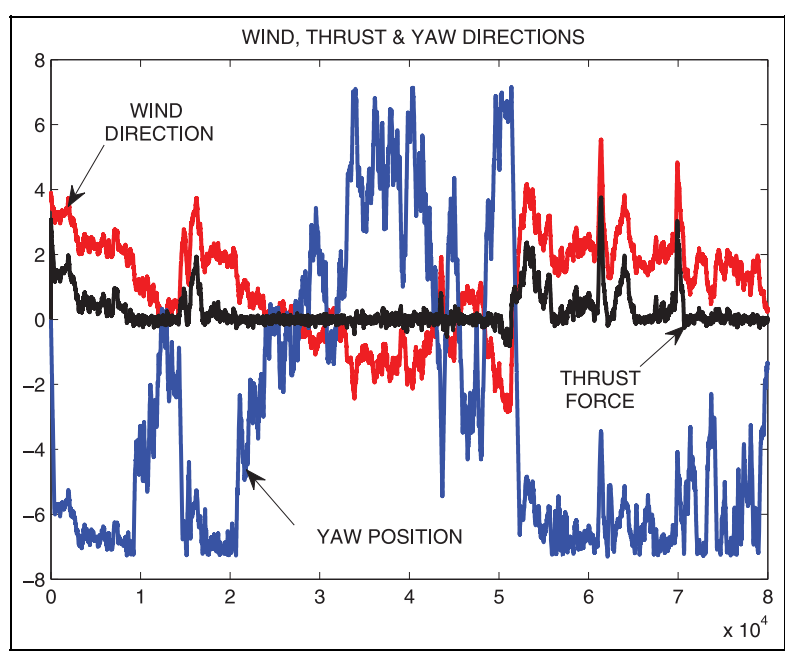

Figure I I. Simulation results of simultaneous speed and yaw turbine control. The control system has the $K \omega^{2}$ turbine speed controller and the thrust force controller. Simulation is performed using the wind direction signal and the wind speed signal measured on the Hönö turbine. The filtered signal of the wind direction is plotted with a red line. The position of the turbine nacelle is plotted with a blue line and the resulting turbine thrust force is plotted with a black line. The heading direction of the vessel is defined as a zero angle. The wind power vector applied to the turbine is projected onto the heading direction of the vessel via control of the yaw angle (see also Figure 10). A proportional regulator provides a satisfactory tracking performance in the presence of the constraints on the yaw angle and the yaw rate. All of the variables are presented with normalized units. Wind speed, turbine speed and tip-speed ratio for this case are plotted in Figure 8.

gyroscopic effects (see Appendix 2) and (b) the range constraint $\bar{\theta}$, which reduces loads.

Performance of the combined control system is illustrated in Figure 11. The filtered signal of the wind direction is plotted with a red line. The position of the turbine nacelle is plotted with a blue line and the resulting turbine thrust force is plotted with a black line. The vector of the thrust force applied to the turbine is projected onto the heading direction of the vessel via control of the yaw angle (see also Figure 10). A proportional regulator provides a satisfactory tracking performance in the presence of the constraints on the yaw rate. Some deviations of the turbine thrust force from the heading direction of the vessel are observed when the turbine yaw angle is saturated.

\section{Conclusion}

A new wind turbine control method is proposed for marine applications, where both turbine speed and thrust force are controllable. A new definition of controllability of the thrust force of the wind turbine was introduced and associated with the invertibility of a nonlinear thrust force model. This allowed application of a new projection algorithm that projects the turbine thrust force onto the heading direction of the vessel, creating a contribution to propulsion despite variations 
in the wind speed and in the wind direction. Coordinated control of turbine speed and thrust minimizes undesirable turbine gyroscopic effects via a proper choice of the algorithm parameters. The results contribute to optimization of the trade-off between fuel savings, due to the turbine powered propulsion, and electricity generation in marine applications.

\section{Acknowledgements}

The author is grateful to Magnus Ellsen for providing measurements from the Hönö wind turbine and to Ola Carlson for managing this project. The author is also grateful to Eric Norelius from Propit, to Christian Haag, Mats Goldberg and Anders Wickström from Scandinavian Wind and to Lars Björkström from Skeab for interesting discussions. In addition, the author is grateful to the anonymous reviewers for their helpful comments.

\section{Declaration of Conflicting Interests}

The author(s) declared no potential conflicts of interest with respect to the research, authorship, and/or publication of this article.

\section{Funding}

The author(s) disclosed receipt of the following financial support for the research, authorship, and/or publication of this article: This work was supported by the Swedish Energy Agency (project number 38919-1).

\section{References}

[1] Bockmann E and Sverre S.Wind turbine propulsion of ships, In: Second international symposium on marine propulsors, Hamburg, Germany, June 2011.

[2] Carlson O and Nilsson P. Development and demonstration of new technology for the use of wind turbines on ships, technical report of the project $N$ 37118-1. Chalmers Publication Library (CPL), 2014.

[3] Carlson O and Nilsson P. Development and demonstration of new technology for the use of wind turbines on ships-phase 2, technical report. Chalmers Publication Library (CPL), 2015.

[4] Pao L and Johnson K.A tutorial on the dynamics and control of wind turbines and wind farms. Proceedings of the American control conference, St. Louis, MO, USA, June 10-12, 2009, pp. 2076-2089.

[5] Stotsky A, Egardt B and Carlson O.An overview of proactive wind turbine control. Energy Sci Eng 2013; 1(1): $1-10$.

[6] Ellsen M and Carlson O. Drift, utveckling och dokumentation vid Chalmers Provstation för vindenergiforskning, Chalmers Technical Report, January 2009 (in Swedish).

[7] Stotsky A.Wind turbine model validation: fusion of simulation and measurement data. Proc IMechE Part I 2014; 228: 734-737.

[8] Johnson K, Pao L, Balas M, Kulkarni V and Fingersh L.Stability analysis of an adaptive torque controller for variable speed wind turbines, NREL Preprint, 2004.
[9] Kim K, Lim C, Oh Y, Kwon I, Yoo N and Paek I.Time-domain dynamic simulation of a wind turbine including yaw motion for power prediction. Int $J$ Precision Eng Manuf 2014; 15: 2199-2203.

[10] Buckspan A, Pao L, Aho J and Fleming P. Stability analysis of a wind turbine active power control system. In: Proceedings of the 2013 American control conference (ACC), Washington, DC, 17-19 June 2013, pp. 14181423.

\section{Appendix I: Stability analysis of $K \omega^{2}$ turbine speed controller}

The combination of equations (8), (9) and (10) results in the closed loop dynamics given by

$$
J \dot{\omega}=\frac{1}{2 N} \rho \pi R^{5} \omega^{2}\left[\frac{C_{p}\left(\lambda, \varphi_{w y}\right)}{\lambda^{3}}-\frac{C_{p m a x}\left(\varphi_{w y}\right)}{\lambda_{*}^{3}}\right]
$$

Taylor series expansion of the power coefficient $C_{p}\left(\lambda, \varphi_{w y}\right)$ around the operating point $C_{\text {pmax }}$ yields

$$
C_{p}\left(\lambda, \varphi_{w y}\right) \approx C_{p \max }\left(\varphi_{w y}\right)+c\left(\lambda-\lambda_{*}\right)^{2}
$$

where $c$ is a negative constant, and the high order terms are neglected. The constant $c$ is associated with the shape of the power coefficient curve around the operating point. This constant can be found using optimization methods for each particular power coefficient curve, and $c \approx-0.02$ for the Hönö turbine. Note that $c$ may depend on the yaw offset. Approximation (18) is valid in a relatively small neighborhood of the operating point $\lambda_{*}$. In particular, for the Hönö turbine, a maximal deviation of approximately fifteen percent from the operating point is allowed for (18) to be valid.

Note that inclusion of the higher-order terms in approximation (18) improves the accuracy of estimation, and enlarges the neighborhood of the operating point. However, the stability analysis gets more complicated in this case.

Then equation (17) can be written as

$$
\begin{aligned}
& J \dot{\omega}=\frac{1}{2 N} \rho \pi R^{5} \omega^{2} C_{p \max }\left(\varphi_{w y}\right)\left[\frac{1}{\lambda^{3}}-\frac{1}{\lambda_{*}^{3}}\right] \\
& +\frac{1}{2 N} \rho \pi R^{5} \omega^{2} \frac{c\left(\lambda-\lambda_{*}\right)^{2}}{\lambda^{3}}
\end{aligned}
$$

Substitution of $\lambda=\frac{\omega R}{V}$ and $\lambda_{*}=\frac{\omega_{d} R}{V}$ and subtraction of $J \dot{\omega}_{d}$ from both sides of (19) result in the error model with respect to the turbine speed mismatch $\omega-\omega_{d}$ given by

$$
\begin{aligned}
& J\left(\dot{\omega}-\dot{\omega}_{d}\right)=\underbrace{-\frac{1}{2 N \lambda_{*}^{3}} \rho \pi R^{5} C_{\text {pmax }}\left(\varphi_{w y}\right) \frac{\left(\omega^{3}-\omega_{d}^{3}\right)}{\omega}}_{\text {stable dynamics }} \\
& +\underbrace{\frac{1}{2 N} \rho \pi R^{4} V c \frac{\left(\omega-\omega_{d}\right)^{2}}{\omega}}_{\text {vanishing term }}-\underbrace{J \dot{\omega}_{d}}_{\text {residual }}
\end{aligned}
$$


This error model has three terms in the right-hand side. The first term represents stable dynamics with respect to the speed mismatch $\omega-\omega_{d}$. This term ensures the convergence of the turbine speed error. The second term vanishes when the turbine speed converges to the desired one. Finally, the third term, which depends on the rate of change of the wind speed, determines a residual error of the system.

Taylor series expansion of the terms $\frac{\left(\omega^{3}-\omega_{d}^{3}\right)}{\omega}, \frac{\left(\omega-\omega_{d}\right)^{2}}{\omega}$ around the operating point $\omega_{d}$

$$
\begin{aligned}
& \frac{\left(\omega^{3}-\omega_{d}^{3}\right)}{\omega} \approx 3 \omega_{d}\left(\omega-\omega_{d}\right) \\
& \frac{\left(\omega-\omega_{d}\right)^{2}}{\omega} \approx \frac{\left(\omega-\omega_{d}\right)^{2}}{\omega_{d}}
\end{aligned}
$$

with subsequent substitution of these terms into the error model (20) yields

$$
\begin{aligned}
J\left(\dot{\omega}-\dot{\omega}_{d}\right)= & -\frac{3}{2 N \lambda_{*}^{2}} \rho \pi R^{4} V C_{p \max }\left(\varphi_{w y}\right)\left(\omega-\omega_{d}\right) \\
& +\frac{1}{2 N \lambda_{*}} \rho \pi R^{5} c\left(\omega-\omega_{d}\right)^{2}-J \dot{\omega}_{d}
\end{aligned}
$$

The system has the following Lyapunov function $Q=\frac{J}{2}\left(\omega-\omega_{d}\right)^{2}$, whose derivative along the solutions of the error model (23) can be written as

$$
\begin{gathered}
\dot{Q} \leqslant\left\{\omega-\omega_{d}\right\}^{2}\left\{-\frac{3}{2 N \lambda_{*}^{2}} \rho \pi R^{4} V C_{p \max }\left(\varphi_{w y}\right)\right. \\
\left.+\frac{1}{2 N \lambda_{*}} \rho \pi R^{5}|c|\left|\omega-\omega_{d}\right|\right\}+c_{1}\left|\omega-\omega_{d}\right|
\end{gathered}
$$

where $c_{1}=J \overline{\dot{\omega}_{d}}$ and $\overline{\dot{\omega}_{d}}$ is an upper bound of $\left|\dot{\omega}_{d}\right|=\left|\frac{\lambda_{*} \dot{V}}{R}\right|$.

For the error model to be stable, the term in the bracket $\{\cdot\}$ that defines the region of attraction for the initial turbine speed mismatch $\left|\omega(0)-\omega_{d}(0)\right|$ should be negative. It implies that $\omega(0)$ should be sufficiently close to $\left.\omega_{d}(0)\right)$ for the system to be stable. Two variables in the bracket $\{\cdot\}$, (a) the wind speed $V$ and (b) power coefficient $C_{\text {pmax }}\left(\varphi_{w y}\right)$, have an impact on the region of attraction and the convergence rate.

The region of attraction has the most simple form in the case of a constant wind speed (for which the system is exponentially stable) and can be written as

$$
\left|\omega(0)-\omega_{d}(0)\right|<\frac{3 C_{\text {pmax }}\left(\varphi_{w y}\right)}{\lambda_{*} R|c|} V
$$

In other words, if the initial turbine speed satisfies inequality (25), then the turbine speed error never leaves this region. Note that the ratio $\frac{3 C_{p \max }\left(\varphi_{w y}\right)}{\lambda_{*} R|c|} \mathrm{rad} / \mathrm{m}$ is the constant of proportionality that relates the initial turbine speed error presented in $\mathrm{rad} / \mathrm{s}$ and the wind speed presented in $\mathrm{m} / \mathrm{s}$. This ratio is close to one for the Hönö turbine and (25) gets the simplest form $\left|\omega(0)-\omega_{d}(0)\right|<V$ in the case of perfect alignment of the turbine rotational axis with the wind (which corresponds to zero yaw angle), where $C_{p m a x}\left(\varphi_{w y}\right)$ reaches its maximal value (see Figure 7 ). The region of attraction is quite large for the Hönö turbine where the rotational radius is small (see Table 1). The region of attraction decreases in size (and the convergence rate deteriorates) when the turbine yaw offset increases. Minimal region of attraction and the worst convergence rate correspond to a yaw angle which is close to $90^{\circ}$. The size of the region increases and the rate of convergence gets higher when the wind speed increases. However, variations in the wind speed increase residual error. Therefore, poor performance of the $K \omega^{2}$ turbine speed controller is expected in the case of large yaw offset (due to controllability problems) and for low and fast varying wind speed (due to the convergence rate deterioration and residual error).

In general, $\varlimsup_{\lim _{t \rightarrow \infty}}\left|\omega(t)-\omega_{d}(t)\right|=\delta$ for some constant $\delta>0$, for time-varying wind speed, which depends on the rate of change of the wind speed. In other words, the closed-loop system is exponentially stable with a residual error. Convergence of the tipspeed ratio $\lambda$ to the desired one $\lambda_{*}$ follows from the convergence of the turbine speed error. This completes the proof.

Note that the proof of stability of the conventional $K \omega^{2}$ turbine speed controller using a linearization technique is also presented in ${ }^{10}$. However, Lyapunov treatment of the nonlinear turbine speed error model presented in this paper provides a more accurate estimate of the region of attraction of the system.

Note also that the yaw offset is accounted for via $C_{p}\left(\lambda, \varphi_{w y}\right)$ only in the turbine rotational dynamics, described by equations (6) and (8). This model, which decouples the speed and yaw motions, is valid for constant (or slowly varying) yaw offset only. A complete model, which considers interactions between speed and yaw motions, should be used for fast-varying yaw offset.

\section{Appendix 2: Yaw rate limitation via constraints on the control action}

The reduced-order model of the DC motor (13), which accounts for the mechanical subsystem only, can be rewritten as

$$
T_{m} \ddot{\theta}+\dot{\theta}=G u_{d c}
$$

The proportional regulator that controls the voltage can be written as

$$
u_{d c}=-k_{p}\left(\theta-\theta_{d}\right)
$$

where $\theta_{d}$ is the desired yaw angle, and $k_{p}$ is a positive constant to be chosen so as to make the closed-loop system (26), (27) stable. Note that the same proportional regulator also makes the full-order system stable (equations (12) and (27)). 
Introduction of a virtual constraint $\bar{u}$ (saturation function) on the input voltage of the DC motor as an additional design parameter where

$$
\left|u_{d c}\right| \leqslant \bar{u}
$$

allows regulation of the upper bound of the turbine yaw rate.

The yaw model (26) can be written in the form

$$
\frac{d}{d t} \dot{\tilde{\theta}}=-\frac{1}{T_{m}} \dot{\tilde{\theta}}+\frac{G}{T_{m}} u_{d c}, \quad\left|u_{d c}\right| \leqslant \bar{u}
$$

where the yaw rate $\dot{\tilde{\theta}}=\dot{\theta}$ is a state variable, assuming that the desired yaw angle is constant.

Notice that the error model (29) represents stable dynamics driven by the input $\frac{G}{T_{m}} u_{d c}$, whose upper bound $\frac{G}{T_{m}} \bar{u}$ can be controlled via a proper choice of $\bar{u}$. Reduction of $\bar{u}$ implies more severe constraints on the yaw rate $\dot{\tilde{\theta}}$.

Solution of (29) obeys the upper bound

$$
|\dot{\tilde{\theta}}(t)| \leqslant \sqrt{\dot{\tilde{\theta}}^{2}(0) e^{-\frac{t}{T_{m}}}+G^{2} \bar{u}^{2}} \leqslant C
$$

which shows that the absolute value of the yaw rate $\tilde{\theta}(t)$ can be limited by a constant $C>0$ via a proper choice of the design parameter $\bar{u}$ after some transient. Indeed, the choice of a sufficiently small upper bound $\bar{u}$ (i.e. $\bar{u} \leqslant \frac{C}{G}$ ) guarantees that the absolute value of the yaw rate is limited by any constant $C$, provided that $\tilde{\theta}(0)=0$. The constant $C$ should be chosen from the interval of $[0.1,1]$ degrees per second, which is small enough to minimize the gyroscopic moment of the turbine.

Note that a similar result can be obtained for the time-varying desired yaw angle $\theta_{d}=\theta_{d}(t)$ with bounded first and the second derivatives. The bounds on the derivatives appear explicitly in the upper bound on the yaw rate, in this case. This imposes constraints on the desired trajectory of the turbine yaw angle for reduction of the turbine gyroscopic effects. This, in turn, implies some additional constraints on the rate of change of the wind speed and of the wind direction, which can be handled by the turbine yaw control system without significant gyroscopic effects. 